\title{
Global Montaigne. Mélanges en l'honneur de Philippe Desan, dir. J. BALSAMO et A. GRAVES
}

\section{Maurizio Busca}

\section{(2) OpenEdition}

\section{Journals}

\section{Edizione digitale}

URL: https://journals.openedition.org/studifrancesi/44928

DOI: 10.4000/studifrancesi.44928

ISSN: 2421-5856

\section{Editore}

Rosenberg \& Sellier

\section{Edizione cartacea}

Data di pubblicazione: 1 août 2021

Paginazione: $363-364$

ISSN: 0039-2944

\section{Notizia bibliografica digitale}

Maurizio Busca, «Global Montaigne. Mélanges en l'honneur de Philippe Desan, dir. J. Balsamo et A. graves», Studi Francesi [Online], 194 (LXV | II) | 2021, online dal 01 septembre 2021, consultato il 15 octobre 2022. URL: http://journals.openedition.org/studifrancesi/44928 ; DOI: https://doi.org/10.4000/ studifrancesi.44928

Questo documento è stato generato automaticamente il 15 octobre 2022

\section{(c) (i) (9)}

Creative Commons - Attribuzione - Non commerciale - Non opere derivate 4.0 Internazionale - CC BY NC-ND 4.0

https://creativecommons.org/licenses/by-nc-nd/4.0/ 


\title{
Global Montaigne. Mélanges en l'honneur de Philippe Desan, dir. J.
} BALSAMO et A. GRAVES

\author{
Maurizio Busca
}

\section{NOTIZIA}

Global Montaigne. Mélanges en l'honneur de Philippe Desan, dir. J. BALSAMO et A. GRAVES, Paris, Classiques Garnier, 2021, pp. 797.

1 Negli ultimi trent'anni, gli studi su Montaigne hanno conosciuto in Philippe Desan il loro più zelante cultore. Autore di una vastissima bibliografia critica sugli Essais e sul loro contesto di elaborazione e diffusione, direttore dei "Montaigne Studies", responsabile di decine di eventi scientifici e programmi di ricerca, Desan ha contribuito in particolare a ricollocare Montaigne e la sua opera nella dimensione della storia sociale e politica. Tale meritorio lavoro è omaggiato dalla presente raccolta, che riunisce quarantotto interventi riconducibili a quattro grandi campi di indagine: la ricostruzione della cornice storica, politica, sociale e culturale nella quale si inserisce Montaigne; l'interpretazione di singoli luoghi testuali o di aspetti che attraversano la sua produzione letteraria; lo studio di diverse forme di «materialità» del testo montaignano, dagli oggetti in esso evocati alle peculiarità tipografiche delle diverse edizioni; infine, l'analisi delle dinamiche della ricezione, dal Cinquecento ad oggi.

2 L'elevato numero dei contributi non consente di presentare il contenuto di ognuno di essi in questa sede; è però doveroso evocare $i$ principali ambiti tematici che attraversano il volume. Un nutrito nucleo di studi prende in esame aspetti della vita e del pensiero di Montaigne relativi alla sfera della socialità: ABROUGUI, DE SOUZA BIRCHAL, GIOCANTI, GONTIER, HOFFMANN, LLINÀS BEGON, PEÑA, RANDALL e SELLEVOLD guardano infatti alla sua attività politica e diplomatica ( $\mathrm{e}$, inversamente, la sua retraite), oltre alle sue riflessioni sulle diverse forme dei legami sociali. Un nucleo altrettanto ricco, quanto a 
consistenza e varietà interna, è costituito dai saggi di AZAR FILHO, FERRER, GIACOMOTTOCHARRA, GRAVES, LANGER, LESTRINGANT, LITSARDAKI, MIERNOWSKI, PAGANINI, PESSELE e MARKOVITS, dedicati alla filosofia - e all'ironia - di Montaigne, non soltanto nella sua declinazione scettica. Un terzo grande nucleo è formato dai lavori di ABECASSIS, CROUZET, DOTOLI, FONTANA, GAO, HAMLIN, MAGNIEN, MIYAKAWA, O'BRIEN, PREDA e TOFTGAARD, che esplorano la dimensione della ricezione di Montaigne in tutta la sua estensione cronologica (dal Cinquecento ai nostri giorni) e geografica (europea ed extraeuropea). L'ambito della religione, sondato da BAYOD, HEITSCH, MACPHAIL e TINGUELY, è anch'esso affrontato da prospettive diverse, che vanno dalle osservazioni sui riti ebraici alle polemiche di argomento teologico, e che spesso si intrecciano con questioni di natura filosofica. BALSAMO, CAVALLINI, CONIHOUT e FRISCH, servendosi degli strumenti della bibliografia materiale, osservano le specificità tipografiche e le pratiche editoriali che caratterizzano diverse edizioni cinque-secentesche degli Essais, mentre BOUTCHER, HUCHON e SMITH illustrano aspetti puntuali dell'esperienza del Montaigne lettore ed editore dei testi di La Boétie. Analisi di ordine linguistico e retorico sul Journal e sugli Essais sono al centro degli interventi di ADAM, DUCHÉ e LEGROS, mentre ROMÃo e SÈVE esaminano le considerazioni di ordine estetico e antropologico formulate da Montaigne negli Essais e nel Voyage en Italie, concernenti sia la pittura e le arti figurative che la musica e gli strumenti musicali.

Benché estremamente sintetici, questi accenni al contenuto dei mélanges offerti a Desan saranno sufficienti per chiarire la scelta del titolo: se Montaigne resta un autore inesauribile, il presente volume permette di avvicinarne la figura e l'opera attraverso una pluralità di prospettive complementari, restituendone un quadro quanto più possibile globale. Il contributi raccolti sono i seguenti.

Première partie («Autour de Montaigne»): George HoffmanN, A Judge That Never Was. Montaigne on the First Extraordinary Chamber, pp. 15-28; Warren BOUTCHER, Writing diversity. Literary objects in Montaigne's "Journal de voyage", pp. 29-49; Frédéric TINGUELY, «De la circoncision». Montaigne et la communauté juive de Rome, pp. 51-61; Thierry GONTIER, La «retraite» de Montaigne. Une position politique?, pp.63-78; Olfa ABROUGUI, Réflexions autour des guerres civiles dans les "Essais" de Montaigne, pp. 79-90; Paul J. SMITH, Montaigne, Amyot et les traductions de Plutarque par La Boétie, pp. 91-102; Mireille HUCHON, Monstrances montaignistes pour reliques boétiennes, pp. 103-116; Peter MACK, Montaigne, Erasmus and the Moriae Encomium, pp. 117-131; Eric MACPHAIL, "On giving the lie". Montaigne, Calvin, and the Death of Michel Servet, pp. 133-142; Jordi BAYOD, Montaigne, Juste Lipse et la justification des écrits «sans meslange de theologie», pp. 143-156. Deuxième partie («Interprétations»): Dorothea HEITSCH, Montaigne archiviste. I, 8: «De l'oisiveté», pp. 159-171; Joan Lluís LLINÀs BEGON, La formation du conseiller du prince dans «De l'institution des enfans», pp. 173-185; Michael RANDALL, On the Appearance of Guy de Brimeu, seigneur d'Humbercourt, in «De la diversion» (III, 4), pp. 187-198; Frank LESTRINGANT, «Plaisants causeurs [!]». "Essais", III, 11, pp. 199-207; Maria LITSARDAKI, Eutopies, dystopies, hétérotopies chez Montaigne, pp. 209-218; Santiago Francisco PEÑA, «Nous sommes part du monde». Montaigne, Greece and Italy, pp. 219-229; Véronique FERRER, La gaieté de Montaigne, pp. 231-242; Nicola PANICHI, La prophétie est morte, vive la prophétie. Le cas Montaigne, pp.243-261; Sylvia GIOCANTI, Montaigne, philosophe du social, pp. 263-277; Telma DE SOUZA BIRCHAL, When women are the issue, is Montaigne still thinking the social?, pp.279-292; Gianni PAGANINI, Pyrrhonism vs Internalism. Montaigne and Sanches, pp. 293-301; Celso M. AZAR FILHO, Sur les rapports entre 
l'ignorance, la science, la philosophie et le scepticisme chez Montaigne, pp. 303-313; Ullrich LANGER, Montaigne, Skepticism and Finitude. Montaigne, Horkheimer: Unhelpful Skepticism in a Limited World, pp. 315-326; Jan MIERNowsKI, Montaigne, Skepticism and Finitude. Montaigne, Meillassoux: Helpful Skepticism and the Multiplicity of Worlds, pp. 327-344. Troisième partie («Matérialités»): Amy GRAVES, Montaigne and Things, pp.347-355; Violaine GIACOMOTTOCHARRA, "C'est moy, c'est mon essence». Un portrait de Montaigne in lumine aristotelis, pp. 357-370; Wolfgang ADAM, «Tout le magasin des mots et des figures». Images et métaphores dans le "Journal de voyage" de Montaigne, pp. 371-385; Bernard SÈVE, L'instrumentarium de Montaigne, pp. 387-400; Rui Bertrand ROMÃo, Le regard et le geste. Considérations sur Montaigne et les peintres, pp. 401-410; Alain LEGROS, «Épékhô, c'est-à-dire je soutiens, je ne bouge». Jeu de paume, histoire et philosophie, pp. 411-423; Kirsti SELLEVoLD, "Je vis en mon enfance». Communication non verbale dans les "Essais" (I, 24: «Divers evenemens de mesme conseil»), pp.425-438; Isabelle DE CONIHOUT, Notes préalables à un recensement des exemplaires remarquables des ceuvres de Montaigne. Les "Essais" de 1580, pp. 439-448; Jean BALSAMO, Quelques remarques pour l'appréciation bibliophilique de l'édition des "Essais" (Paris, L'Angelier, 1588), pp. 449-464; Véronique DUCHÉ, "Certes, je lis cet autheur avec un peu plus de reverence et de respect» (II, 10). The "Essais" and corpus linguistics, pp. 465-486; Concetta CAVALLINI, Montaigne entre parenthèses. ("Essais" 1582, livre II), pp. 487-501; Andrea FRISCH, Pointing fingers. Indexical tables in the "Essais" (Simon Goulart, Abel L'Angelier, and Henry $V$ Estienne), pp. 503-515. Quatrième partie («Réception»): Shinya MIYAKAWA, Montaigne et Tchouang-tseu (Zhuangzi). Deux penseurs face à la mythologie, pp. 519-535; John o'BRIEN, Self before the self. The evidence of early readers of the "Essais", pp. 537-548; William M. HAMLIN, On Florio's "Repentance", pp. 549-559; Giovanni DoTOLI, Montaigne-Pascal. Nouvelle lecture, pp. 561-575; André PESSEL ? et Francine MARKOVITS, Montaigne et la culture de l'exemplarité au XVII eiècle, pp. 577-596; Michel MAGNIEN, Les "Essais dans le goût de ceux de Montagne, composés en 1736" de René-Louis d'Argenson, pp. 597-614; Alessandra PREDA, Traduire Montaigne au XVIII siècle. Les "Saggi" de Giulio Perini, pp. 615-630; Biancamaria FONTANA, Michel de Montaigne, Germaine de Staël and the writer's ambition. "De l'influence des passions" and the "Essais", pp. 631-643; Denis CROUZET, Auguste Alexis Floréal Baron ou le "bon sens» de Montaigne dans l'historicisation de l'histoire de la littérature française, pp. 645-685; Anders TOFTGAARD, A Danish friend of Montaigne. Frederik Thorkelin (1904-1997) and his Bibliotheca Thorkeliniana, pp. 687-705; Ji GAO, Quelques thèmes récurrents dans la représentation de Montaigne en Chine durant l'ère républicaine (1912-1949), pp. 707-721; Jack I. ABECASSIS, La peau e[s]t le masque. Lire Montaigne avec Clément Rosset, pp. 723-734. 